\title{
Trombectomia mecânica no acidente vascular cerebral isquêmico agudo: revisão de literatura
}

\author{
Mechanical thrombectomy in acute ischemic stroke: literature review
}

\begin{abstract}
Ariane Emy Sato Rodrigues Alves ${ }^{1}$, Brigite Cunha Cese ${ }^{1}$, Carlos Felipe de Oliveira Reyna ${ }^{1}$, Jessica Ferreira de Oliveira ${ }^{1}$, Julianna Sayuri Kaneko ${ }^{1}$, Marcella Sampaio Gouveia ${ }^{1}$, Matheus Fischer Severo Cruz Homem ${ }^{1}$, Rafael Garcia de Oliveira ${ }^{1}$, Vivian Dias Baptista Gagliardi' ${ }^{2}$, Sandra Regina Schwarzwalder Sprovieri ${ }^{3}$
\end{abstract}

\section{Resumo}

Objetivo: O objetivo deste trabalho é avaliar a evidência científica relacionada ao tratamento endovascular do Acidente Vascular Cerebral (AVC) isquêmico através da trombectomia mecânica. Métodos: Trata-se de revisão da literatura, utilizando-se os descritores "trombectomia" ou "trombólise mecânica" e "acidente vascular cerebral". Resultados: Dezenove artigos sobre o assunto foram analisados, sendo que destes 8 foram estudos clínicos, observando que o tratamento endovascular do AVC com os dispositivos de $2^{a}$ geração (principalmente os stent retrievers) obtiveram melhor desfecho clínico, avaliado pela escala Rankin modificada, e menor mortalidade em 30 ou 90 dias, sem aumento das taxas de complicações relacionadas à revascularização. Conclusões: A evidência do tratamento endovascular por trombectomia mecânica no AVC isquêmico agudo é recente, por meio de estudos com resultados promissores, mostrando que esta modalidade de tratamento está modificando a história natural dos casos em que há oclusão proximal da circulação cerebral anterior, geralmente mais grave, por apresentarem maior área de isquemia e pior prognóstico. Já estão em andamento estudos que avaliam o benefício da trombectomia expandindo-se o período de tempo hábil para o tratamento do AVC isquêmico agudo em casos selecionados. Ainda, identificamos a necessidade de estudos que utilizem a trombectomia mecânica em outros territórios de irrigação arterial, avaliando-se a viabilidade e os benefícios da trom-

1. Acadêmico da Faculdade de Ciências Médicas da Santa Casa de São Paulo - $6^{\circ}$ Ano do Curso de Graduação em Medicina

2. Médica Assistente da Irmandade da Santa Casa de Misericórdia de São Paulo - Departamento de Clínica Médica

3. Professora Adjunta da Faculdade de Ciências Médicas da Santa Casa de São Paulo - Departamento de Clínica Médica

Trabalho realizado: Faculdade de Ciências Médicas da Santa Casa de São Paulo - Departamento de Clínica Médica. Disciplina de Emergências em Clínica Médica

Endereço para correspondência: Ariane Emy S. R. Alves. Rua Capivari Mirim, 45, Jardim Dracena - 05528-290 - São Paulo-SP

-Brasil.E-mail: ariane.alves.ismart@gmail.com bectomia mecânica no tratamento de oclusões da circulação posterior e de oclusões artérias distais.

Descritores: Trombectomia, Acidente vascular cerebral, Isquemia encefálica, Trombólise mecânica

\begin{abstract}
Objective: The aim of this study is to evaluate the scientific evidence related to the endovascular treatment of ischemic stroke through mechanical thrombectomy. Methods: This paper is a literature review, using the keywords "thrombectomy" or "mechanical thrombolysis" and "stroke". Results: Nineteen articles on the subject were analyzed of which eight were clinical studies remarking that the endovascular treatment of stroke with second-generation devices (mainly stent retrievers) had better clinical outcomes evaluated by the modified Rankin scale, and lower mortality in 30 or 90 days without increasing complication rates related to revascularization. Conclusions: The evidence of endovascular treatment by mechanical thrombectomy in acute ischemic stroke is recent, through studies with promising results showing that this modality of treatment is modifying the natural history of cases where there is proximal occlusion of the anterior cerebral circulation, because of their greater ischemic area and worse prognosis. Studies that evaluate thrombectomy benefits are already underway expanding time for treatment beginning in acute ischemic stroke in selected cases. Furthermore we identified the need for studies that use mechanical thrombectomy in other territories of arterial irrigation, evaluating the feasibility and benefits of mechanical thrombectomy in the treatment of occlusions of the posterior circulation and distal artery occlusions.
\end{abstract}

Keywords: Thrombectomy, Stroke, Brain ischemia, Mechanical thrombolysis

\section{Introdução}

As doenças do aparelho circulatório são a causa mais comum de mortalidade no Brasil, superando 
Alves AESR, Cese BC, Reyna CFO, Oliveira JF, Kaneko JS, Gouveia MS, Homem MFSC, Oliveira RG, Gagliardi VDB, Sprovieri SRS. Trombectomia mecânica no acidente vascular cerebral isquêmico agudo: revisão de literatura. Arq Med Hosp Fac Cienc Med Santa Casa São Paulo. 2018;63(2):110-4.

as causas neoplásicas. As doenças cerebrovasculares ocupam o segundo lugar em mortalidade, logo após as doenças isquêmicas do coração ${ }^{(1)}$.

Deste modo, as doenças cerebrovasculares ocupam enorme importância em saúde pública, por apresentarem alta taxa de morbimortalidade, além dos elevados custos despendidos no tratamento, na reabilitação e no seguimento dos pacientes.

$\mathrm{O}$ tratamento do AVC na fase aguda abrange três etapas:

1. terapia para recanalização, através da trombólise química intravenosa com Ativador do Plasminogênio Tecidual recombinante (rt-PA - recombinant tissue plasminogen activator, ou alteplase), ou trombólise química intra-arterial com rt-PA, ou trombectomia mecânica/tratamento endovascular ${ }^{(2)}$;

2. terapia para evitar lesão secundária: controle rigoroso de glicemia, temperatura corpórea, pressão arterial, oximetria.

3. terapia para aumentar a circulação colateral (terapias com o objetivo de recuperar a área de penumbra estão em estudo, porém ainda faltam medidas com eficácia e segurança comprovadas). Rha e Saver ${ }^{(3)}$, em 2017, publicaram metanálise comparando a evolução natural do AVC (sem tratamento) com diferentes formas de reperfusão. Demonstraram que a recanalização foi o fator preditor independente mais importante para bom prognóstico, com forte associação à independência funcional e a menor mortalidade.

\section{Trombectomia Mecânica (tratamento endovascular)}

A trombectomia mecânica é uma opção para recanalização quando há oclusão proximal de artérias da circulação anterior. Técnica bastante recente, os primeiros estudos publicados em 2013 (SYNTHESIS, MS III, MR RESCUE) utilizavam dispositivos de primeira geração sem ou em associação a alteplase endovenosa, quando em tempo hábil, obtendo como resultado taxas de recanalização de $25 \%$ a $41 \%{ }^{(2,4-5)}$. Já os estudos publicados em 2015 (MR CLEAN, ESCAPE, SWIFT PRIME, EXTEND-IA, REVASCAT) utilizando dispositivos de segunda geração, obtiveram taxas de recanalização entre $59 \%$ a $86 \%$, sendo que os stents retrievers se mostraram mais eficazes, e por isso a AHA/ ASA recomenda que os stents retrievers sejam preferidos em detrimento de outros tipos de dispositivos ${ }^{(6-11)}$.

A tabela 1, apresenta os resultados encontrados nos estudos analisados ${ }^{(4-11)}$ destacando o melhor desfecho clínico nos grupos que realizaram a trombectomia mecânica, avaliado pelo mRS (escala de Rankin modificada ${ }^{(3)}$ - que avalia o grau de independência funcional pós AVC) após 3 meses. Também nota-se que não há diferença significativa entre as taxas de mortalidade

\section{Tabela 1}

Resultados publicados pelos estudos analisados ${ }^{(4-11)}$ em relação à taxa de recanalização, taxas de mRS entre 0 e 2 após 90 dias, mortalidade após 30/90 dias, a mediana de valores das escalas de NIHSS e ASPECTS do paciente na entrada e o dispositivo(s) utilizados pelos estudos.

\begin{tabular}{|c|c|c|c|c|c|c|c|c|}
\hline & \multicolumn{2}{|c|}{ SYNTHESIS } & \multicolumn{2}{|c|}{ IMS III } & \multicolumn{2}{|c|}{ MR RESCUE } & \multicolumn{2}{|c|}{$M R C L E A N$} \\
\hline & Int & Cont & Int & Cont & Int & Cont & Int & Cont \\
\hline Dispositivo & \multicolumn{2}{|c|}{$14 \%$ sten ret } & \multicolumn{2}{|c|}{$1,5 \%$ stent ret } & \multicolumn{2}{|c|}{ Merci ou Penumbra } & \multicolumn{2}{|c|}{ Stent Retrieval } \\
\hline NIHSS na entrada & $13(9-17)$ & $13(9-18)$ & $17-(7-40)$ & $16(8-30)$ & $16-19$ & $16-20$ & $17(14-21)$ & $18(14-22)$ \\
\hline ASPECTS na entrada & & & & & & & $9(7-10)$ & $9(8-10)$ \\
\hline Recanalização & \multicolumn{2}{|c|}{ não publicado } & \multicolumn{2}{|l|}{$41 \%$} & \multicolumn{2}{|l|}{$25 \%$} & \multicolumn{2}{|l|}{$58.7 \%$} \\
\hline $\mathrm{mRS}$ & $30.4 \%$ & $34.8 \%$ & $42.7 \%$ & $40 \%$ & $18.75 \%$ & $20.3 \%$ & $32.6 \%$ & $19.1 \%$ \\
\hline \multirow[t]{3}{*}{ Mortalidade } & $8 \%$ & $6 \%$ & $19.1 \%$ & $21.6 \%$ & $18.75 \%$ & $24 \%$ & $18.9 \%$ & $18.4 \%$ \\
\hline & \multicolumn{2}{|c|}{ ESCAPE } & \multicolumn{2}{|c|}{ SWIFT PRIME } & \multicolumn{2}{|c|}{ EXTEND-IA } & \multicolumn{2}{|c|}{ REVACAT } \\
\hline & Int & Cont & Int & Cont & Int & Cont & Int & Cont \\
\hline Dispositivo & \multicolumn{2}{|c|}{ Solitaire Stent Ret } & \multicolumn{2}{|c|}{ Solitaire Stent Ret } & \multicolumn{2}{|c|}{ Solitaire Stent Ret } & \multicolumn{2}{|c|}{ Solitaire Stent Ret } \\
\hline NIHSS na entrada & $16(13-20)$ & $17(12-20)$ & $17(13-20)$ & $17(13-19)$ & $17(13-20)$ & $13(9-19)$ & $17(14-20)$ & $17(12-19)$ \\
\hline ASPECTS na entrada & $9(8-10)$ & $9(8-10)$ & $9(7-10)$ & $9(8-10)$ & & & $7(6-9)$ & $8(6-9)$ \\
\hline Recanalização & $72.4 \%$ & & $83 \%$ & $40 \%$ & $86 \%$ & & $65.7 \%$ & \\
\hline $\mathrm{mRS}$ & $53 \%$ & $29.3 \%$ & $60 \%$ & $35 \%$ & $71 \%$ & $40 \%$ & $43.7 \%$ & $28.2 \%$ \\
\hline Mortalidade & $10.4 \%$ & $19 \%$ & $9 \%$ & $12 \%$ & $9 \%$ & $20 \%$ & $18.4 \%$ & 15.5 \\
\hline
\end{tabular}

Int= intervenção ; cont= controle; stent ret=stent retriever; $\mathrm{mRS}=$ modified Rankin Scale; NIHSS= NIH Stroke Scale $/$ Score; ASPECTS= Alberta Stroke Program Early CT Score 
Alves AESR, Cese BC, Reyna CFO, Oliveira JF, Kaneko JS, Gouveia MS, Homem MFSC, Oliveira RG, Gagliardi VDB, Sprovieri SRS. Trombectomia mecânica no acidente vascular cerebral isquêmico agudo: revisão de literatura. Arq Med Hosp Fac Cienc Med Santa Casa São Paulo. 2018;63(2):110-4.

após 30 ou 90 dias entre os grupos controle, no qual realizaram apenas tratamento com rt-PA ou manejo para evitar lesão secundária, em relação ao grupo que realizou a trombectomia mecânica sem ou em associação com rt-PA endovenoso.

Os critérios de inclusão utilizados pelos estudos apresentaram algumas variações, mas os atualmente aceitos pela AHA/ $\mathrm{ASA}^{12}$ são:

- Diagnóstico clínico de AVC com déficit calculado pelo NIHSS de 6 ou mais e pontuação ASPECTS de 6 ou mais na TC de crânio sem contraste;

- Tomografia ou ressonância magnética de crânio excluindo hemorragia intracraniana;

- Oclusão arterial proximal da circulação anterior (a. carótida interna na sua porção intracraniana distal ou a. cerebral média em seu segmento proximal, M1) demonstrada por angio-tomografia, angio-ressonância ou na angiografia de subtração digital;

- Ausência de incapacidade importante pré-AVC, definido como escore mRS de 0 ou 1 pré-AVC;

- Idade maior ou igual a 18 anos;

- Início da trombectomia mecânica em até 6 horas do início dos sintomas;

- Obtenção do consentimento Informado

Assim como no tratamento com trombólise química endovenosa, para realizar este procedimento deve-se atingir pressão arterial sistólica menor que $185 \mathrm{mmHg}$ e diastólica menor que $110 \mathrm{mmHg}$, além do controle da glicemia capilar visando a obtenção de valores normais, para reduzir o risco de complicações; também é contraindicado o procedimento na evidência laboratorial de alteração da coagulação (plaquetas $<40$ mil e/ ou INR $>3)^{(12)}$.

É importante lembrar que o uso da alteplase na dose padrão não aumenta risco de sangramento durante ou após o procedimento, devendo ser realizada sempre que o paciente estiver em tempo hábil e não possuir contraindicações à rt-PA, mesmo se estiver sendo considerada a trombectomia mecânica; esta, quando indicada, deve ser realizada assim que possível (após o término da rt-PA), não sendo necessário observar a resposta clínica do paciente após a rt-PA endovenosa, uma vez que a eficácia da trombectomia mecânica em relação à trombólise química nesses casos foi comprovada desde que em tempo hábil ${ }^{(12)}$.

A AHA / ASA $^{(12)}$ considera que apesar da incerteza dos benefícios, em pacientes cuidadosamente selecionados, o uso da trombectomia mecânica com stent retrievers é razoável em pacientes com oclusão das porções M2 e M3 das artérias cerebrais médias, das artérias cerebrais anteriores, das artérias vertebrais, da artéria basilar e das artérias cerebrais posteriores. Além disso, reforça a importância da redução do tempo do início dos sintomas até a reperfusão, pois quanto menor o tempo até a reperfusão melhor o resultado clínico ${ }^{(12)}$

Como os estudos não incluíram pacientes menores de 18 anos não existe comprovação do benefício desde tratamento nessa faixa etária, porém é razoável estender o resultado dos estudos para este grupo etário ${ }^{(12)}$.

Para não retardar o início do tratamento e agilizar o reconhecimento da oclusão proximal de artérias da circulação anterior é recomendado que, durante a realização de exame de imagem o radiologista ou técnico assim que observe a ausência de hemorragia, encaminhe o paciente para realizar a angio-tomografia, angio-ressonância ou angiografia enquanto ainda se encontra no setor de neuroimagem ${ }^{(13)}$.

O objetivo da trombectomia mecânica é a recanalização, que é quantificada pela escala TICI (TICI - thrombolysis in cerebral infarction score $)^{(14)}$. A reperfusão é definida como obtenção de graus TICI 2 b ou 3 após trombólise, nos quais se observa o preenchimento completo do território vascular após o ponto de obstrução, porém com fluxo lentificado no grau TICI 2b, ou normalizado no TICI $3^{(14)}$.

Existem 3 tipos de dispositivos atualmente: 1. Coil retriever: são fios em espiral que englobam o êmbolo permitindo a remoção destes; 2 . Aspiration device: são dispositivos que promovem uma pressão negativa para remover o êmbolo; 3. Stent retrievers: são stents que abertos permitem a adesão do êmbolo à trama do dispositivo permitindo a remoção do mesmo ${ }^{(15)}$.

As principais complicações relacionadas ao procedimento são: hemorragia intracerebral, hemorragia subaracnóidea, microembolizações distais, sangramento sistêmico, sangramento no local de inserção do cateter, formação de aneurisma no local de inserção do cateter, infecção no local de inserção do cateter, morte. Não há evidência de aumento nas taxas de complicações após reperfusão por trombectomia mecânica em relação ao tratamento com rt-PA endovenosa, mesmo quando associada a esta, encontrando taxas semelhantes de complicações, como observamos na Tabela $2^{(4-11)}$.

O estudo REVASCAT também analisou as taxas de complicações relacionadas especificamente ao cateterismo e à trombectomia mecânica observando taxas de $4.9 \%$ de embolização distal, $3.9 \%$ de dissecção arterial, $4.9 \%$ de perfuração arterial, $10.7 \%$ de hematoma no local da punção, $1 \%$ de formação de pseudoaneurisma no local de punção e vasoespasmo necessitando de intervenção em $3.9 \%$ dos pacientes ${ }^{(8)}$.

Pacientes que se encontrem em centros não-especializados para a realização de trombectomia e com critérios para rt-PA endovenosa devem iniciar sua infusão e, caso se identifiquem sinais de oclusão da circulação anterior proximal, devem ser encaminhados para centros especializados em AVC que realizem a trombectomia mecânica, desde que em tempo hábil; 
Alves AESR, Cese BC, Reyna CFO, Oliveira JF, Kaneko JS, Gouveia MS, Homem MFSC, Oliveira RG, Gagliardi VDB, Sprovieri SRS. Trombectomia mecânica no acidente vascular cerebral isquêmico agudo: revisão de literatura. Arq Med Hosp Fac Cienc Med Santa Casa São Paulo. 2018;63(2):110-4.

Tabela 2

Porcentagens das complicações nos grupos controle e nos grupos intervenção por trombectomia mecânica encontradas nos estudos analisados ${ }^{(4-1)}$.

\begin{tabular}{|c|c|c|c|c|c|c|c|c|c|c|c|c|c|c|c|c|}
\hline & \multicolumn{2}{|c|}{ SYNTHESIS } & \multicolumn{2}{|c|}{ IMS III } & \multicolumn{2}{|c|}{ MR RESCUE } & \multicolumn{2}{|c|}{ MR CLEAN } & \multicolumn{2}{|c|}{ ESCAPE } & \multicolumn{2}{|c|}{ SWIFT PRIME } & \multicolumn{2}{|c|}{ EXTEND-IA } & \multicolumn{2}{|c|}{ REVASCAT } \\
\hline & Int & Cont & Int & Cont & Int & Cont & Int & Cont & Int & Cont & Int & Cont & Int & Cont & Int & Cont \\
\hline Deterir. neurol. & $9 \%$ & $7 \%$ & & & & & & & & & & & & & $15.5 \%$ & $12.6 \%$ \\
\hline Morte em 7d & $8 \%$ & $6 \%$ & $15 \%$ & $10.8 \%$ & & & $11.6 \%$ & $12.4 \%$ & & & & & & & $937 \%$ & $4.9 \%$ \\
\hline Morte em 30-90d & & & $19.1 \%$ & $21.6 \%$ & $18.75 \%$ & $24 \%$ & $18.9 \%$ & $18.4 \%$ & $10.4 \%$ & $19 \%$ & $9 \%$ & $12 \%$ & $9 \%$ & $20 \%$ & $18.4 \%$ & $15.5 \%$ \\
\hline Hemor. Intracr. & $6 \%$ & $6 \%$ & $6.2 \%$ & $5.9 \%$ & $9 \%$ & $6 \%$ & $7.7 \%$ & $6.4 \%$ & $3.6 \%$ & $2.7 \%$ & $0 \%$ & $3 \%$ & $0 \%$ & $6 \%$ & $1.9 \%$ & $1.9 \%$ \\
\hline Hemor. Subar. & & & $11.5 \%$ & $5.8 \%$ & & & $0.9 \%$ & $0 \%$ & & & $4 \%$ & $1 \%$ & & & $4.9 \%$ & $1.9 \%$ \\
\hline AVCi recorrente & $2 \%$ & $2 \%$ & $5.1 \%$ & $6.3 \%$ & & & $5.6 \%$ & $0.4 \%$ & & & & & & & $3.9 \%$ & $2.9 \%$ \\
\hline Rela. Procedim. & & & $16.1 \%$ & & & & & & $2.4 \%$ & & & & & & & \\
\hline
\end{tabular}

Int = intervenção; cont = controle; deter. neurol.= deterioração neurológica; hemor. intracr.= hemorragia intracraniana; hemor. subar.= hemorragia subaracnóide; $\mathrm{AVCi}=$ acidente vascular cerebral isquêmico; relac. procedim. $=$ relacionados ao procedimento.

a chamada estratégia "Drip and Ship" (14). Apenas $10 \%$ dos pacientes com AVC isquêmico tem oclusão proximal da circulação anterior, e portanto a maioria não tem indicação deste tratamento; porém, a terapia endovascular vem mudando o desfecho clínico deste grupo de pacientes, que em geral evoluíram com áreas isquêmicas grandes, que resulta em tempo prolongado de internação, pior grau de independência e maiores taxas de mortalidade ${ }^{(14)}$.

\section{Trombectomia Mecânica no Brasil}

No Brasil, os dispositivos para trombectomia aprovados pela ANVISA são: os "Aspiration devices" (Penumbra) e os "Stents retrievers" (Solitaire e Trevo). O dispositivo MERCI ("coil retriever") não está disponível no Brasil, uma vez que não estão disponíveis estudos clínicos randomizados com dados de prognóstico utilizando este tipo de dispositivo (2).

Apesar da aprovação desses dispositivos em território brasileiro, este tipo de terapêutica ainda não está disponível no setor público de saúde. O estudo Resilient, iniciado em janeiro de 2017, é um ensaio clínico randomizado que está sendo realizado pelo Ministério da Saúde (DECIT/SAS) em parceria com o $\mathrm{CNPq}$, com o intuito de comprovar os resultados internacionais em âmbito nacional. Este estudo avalia se os hospitais do sistema público de saúde no Brasil apresentam estrutura organizacional suficiente para aplicar esta terapêutica além de avaliar se estes locais atingem os mesmos resultados de eficácia e segurança dos comprovados pelos estudos internacionais, para posterior implementação da terapia endovascular no SUS. Este estudo analisa se a trombectomia mecânica com stent-retriever e/ou tromboaspiração é superior ao tratamento clínico em atingir evolução neurológica mais favorável em 90 dias em casos de AVC isquêmico agudo com oclusão de vasos da circulação anterior proximal em pacientes atendidos no sistema público de saúde ${ }^{(16)}$.
De acordo com as "Diretrizes brasileiras para o tratamento endovascular de pacientes com acidente vascular isquêmico agudo" de janeiro de 2017, o sucesso da implementação do tratamento endovascular nos serviços públicos e privados depende de planejamento e organização de recursos locais e da organização de uma rede de AVC capaz de identificar e encaminhar os pacientes para centros especializados. Nessa diretriz, as recomendações para a realização da trombectomia seguem os critérios já relatados neste trabalho ${ }^{(17)}$.

\section{Perspectivas para o futuro}

Considerando as limitações dos estudos atuais, temos como perspectivas para o futuro o estudo dos efeitos da trombectomia mecânica na circulação posterior do sistema nervoso central e também em oclusões distais.

Ainda dentro das perspectivas futuras, o estudo DAWN ${ }^{(18)}$ observou benefício da utilização da trombectomia mecânica em pacientes com até 24 horas do início dos sintomas ${ }^{(18)}$. Neste estudo foram selecionados pacientes com oclusão de grandes vasos apresentando início de sintomas entre 6 e 24 horas (média 13 horas) e que apresentassem um pequeno núcleo de infarto em relação ao escore de déficits apresentados (calculado pelo National Institutes of Health Stroke Scale- NIHSS) - chamado de mismatch entre o déficit e a área de infarto. Seus resultados demonstraram que a trombectomia mecânica, realizada conforme o protocolo do estudo, se manteve benéfica até as 24 horas desde a última vez que o paciente foi visto sem sintomas, e confirmou que o quão mais precoce o tratamento, maiores as chances do paciente apresentar independência funcional ${ }^{(18)}$. Os resultados mostraram melhora de independência funcional e de sequelas em relação aos controles ${ }^{(18)}$.

Desta maneira, como observado no estudo DAWN ${ }^{(18)}$ e a ser verificado em demais estudos futuros 
Alves AESR, Cese BC, Reyna CFO, Oliveira JF, Kaneko JS, Gouveia MS, Homem MFSC, Oliveira RG, Gagliardi VDB, Sprovieri SRS. Trombectomia mecânica no acidente vascular cerebral isquêmico agudo: revisão de literatura. Arq Med Hosp Fac Cienc Med Santa Casa São Paulo. 2018;63(2):110-4.

(DEFUSE-3, possível MR CLEAN LATE) é possível que ocorra mudança do tempo hábil de realização do tratamento endovascular de oclusões arteriais, a depender da seleção dos pacientes ${ }^{(19)}$.

\section{Conclusão}

Apesar de apenas $10 \%$ dos pacientes com AVC isquêmico terem alguma oclusão proximal da circulação anterior, a trombectomia mecânica vem mudando o desfecho clínico deste grupo de pacientes, cujo quadro está associado a grande morbi-mortalidade. Já existem estudos em andamento que objetivam avaliar o benefício da trombectomia expandindo o tempo hábil para seu tratamento. Identificamos ainda a necessidade de novos estudos que avaliem a viabilidade e os benefícios da trombectomia em oclusões distais e em oclusões da circulação posterior.

\section{Referências}

1. Brasil. Ministério da saúde. Informações de saúde TABNETEstatísticas vitais. DATASUS. [online]. Disponível em: http:/ / tabnet.datasus.gov.br (14 set 2017)

2. Martins SCO, Freitas GR, Pontes-Neto OM, Pieri A, Moro CEC, Jesus PAP, et al. Guidelines for acute ischemic stroke treatment: part II:stroke treatment. Arq Neuro-Psiquiatr. 2012; 70(11):88593.

3. Rha J, Saver JL. The impact of the recanalization on ischemic stroke outcome: a meta-analysis. Stroke. 2017; 38(3):967-73.

4. Kidwell CS, Jahan R, Gornbein J, Alger JR, Nenov V, Ajani Z, et al. A trial of imaging selection and endovascular treatment for ischemic stroke. N Engl J Med. 2013; 368(10):914-23.

5. Broderick JP, Palesch YY, Demchuk AM, Yeatts SD, Khatri P, Hill $\mathrm{MD}$, et al. Endovascular therapy after Intravenous t-PA versus t-PA alone for stroke. N Engl J Med. 2013; 368(10):893-903.

6. Ciccone A, Valvassori L, Nichelatti M, Sgoifo A, Ponzio M, Sterzi $\mathrm{R}$, et al. Endovascular treatment for acute ischemic stroke. $\mathrm{N}$ Engl J Med. 2013; 368(10):904-13.

7. Goyal M, Demchuk AM, Menon BK, Eesa M, Rempel JL, Thornton J, et al. Randomized assessment of rapid endovascular treatment of ischemic stroke. N Engl J Med. 2015; 372(11):1019-30.

8. Jovin TG, Chamorro A, Cobo E, Miquel MA, Molina CA, Rovira A, et al. Thrombectomy within 8 hours after symptom onset in ischemic stroke. N Engl J Med. 2015; 372(24):2296-306.

9. Campbell BCV, Mitchell PJ, Kleinig TJ, Dewey HM, Churilov L, Yassi N, et al. Endovascular therapy for ischemic stroke with perfusion-imaging selection. N Engl J Med. 2015; 372(11):100918.
10. Saver JL, Goyal M, Bonafe A, Diener HC, Levy EI, Pereira VM, et al. Stent-Retriever thrombectomy after intravenous t-PA vs. t-PA alone in stroke. N Engl J Med. 2015; 372(24):2285-95.

11. Berkhemer OA, Fransen PSS, Beumer D, van den Berg LA, Lingsma HF, Yoo AJ, et al. A randomized trial of intraarterial treatment for acute ischemic stroke. N Engl J Med. 2015; 372(1):11-20.

12. American Heart Association. AHA/ASA Guideline. 2015 AHA/ASA focused update of the 2013 guidelines for the early management of patients with acute ischemic stroke regarding endovascular treatment. A Guideline for healthcare professionals from the American Heart Association/American Stroke Association. [online]. Available from: http:/ / stroke.ahajournals. org/content/early/2015/06/26/STR.00000000000000074 (2017 Oct 1 )

13. Campbell BCV, Donnan GA, Mitchell PJ, Davis SM. Endovascular thrombectomy for stroke: current best practice and future goals. Stroke Vasc Neurol. 2016;1(1):16-22.

14. Oliveira Filho J, Samuels OB. Approach to reperfusion therapy for acute ischemic stroke. UpToDate. [online]. Literature review current through: Feb 2018. | This topic last updated: Mar 12, 2018. Available from: https://www.uptodate.com/contents/ reperfusion-therapy-for-acute-ischemic-stroke?source=search result\&search=trombectomia $\% 20 \mathrm{mec} \% \mathrm{C} 3 \% \mathrm{~A} 2$ nica\&selectedT itle=1 22\#H28 (2017 Oct 11)

15. Raychev R, Saver JL. Mechanical thrombectomy devices for treatment of stroke. Neurol Clin Pract. 2012; 2(3):231-235.

16. Martins SCO, Nogueira R, Carvalho JJF, Montalverne J, Pontes-Neto OM, Abud D, et al. Randomization of endovascular treatment with stent-retriever and/or thromboaspiration vs. best medical therapy in acute ischemic stroke due to large vessel occlusion trial (RESILIENT). Clinical Trial. [online]. Available from: https: / clinicaltrials.gov/ct2/show/NCT02216643 (2017 Sept 15).

17. Pontes-Neto OM, Cougo P, Martins SCO, Abud DG, Nogueira RG, Miranda M, et al. Diretrizes brasileiras para o tratamento endovascular de pacientes com acidente vascular isquêmico agudo. Arq Neuropsiquiatr. 2017;75(1):50-6.

18. Nogueira RG, Jadhav AP, Haussen DC, Bonafe A, Budzik RF, et al. Thrombectomy 6 to 24 hours after stroke with a mismatch between deficit and infarct. N Engl J Med. 2018; 378(1):11-21.

19. Law LY, Campbell BCV, Wijeratne T. Advances in endovascular therapy for ischemic stroke: a whole new ball game. Neurol Clin Pract. 2016; 6(1):49-54.

Trabalho recebido: $16 / 01 / 2018$

Trabalho aprovado: 18/07/2018 